\title{
Barriers to and facilitators for using nutrition apps: a scoping review and conceptual framework
}

\author{
Laura Maria König, Christiane Attig, Thomas Franke, Britta Renner \\ Submitted to: JMIR mHealth and uHealth \\ on: May 12, 2020
}

Disclaimer: (C) The authors. All rights reserved. This is a privileged document currently under peer-review/community review. Authors have provided JMIR Publications with an exclusive license to publish this preprint on it's website for review purposes only. While the final peer-reviewed paper may be licensed under a CC BY license on publication, at this stage authors and publisher expressively prohibit redistribution of this draft paper other than for review purposes. 


\section{Table of Contents}

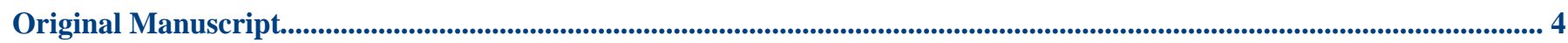

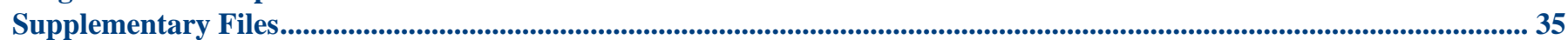

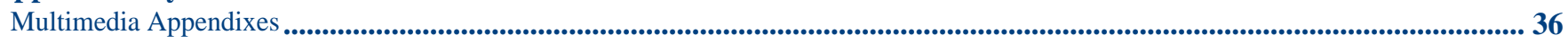

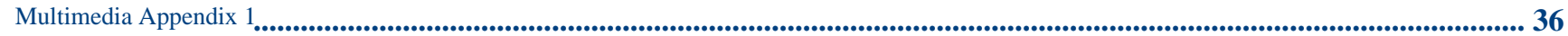

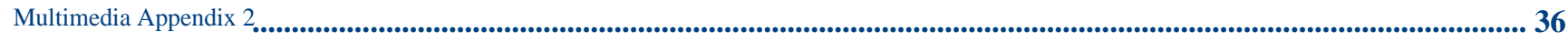

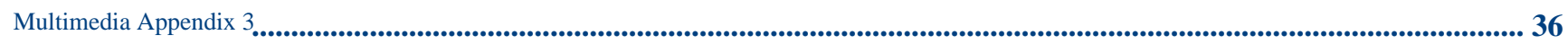




\title{
Barriers to and facilitators for using nutrition apps: a scoping review and conceptual framework
}

\author{
Laura Maria KönigBSc, MSc, PhD, ; Christiane AttigBSc, MSc, ; Thomas FrankePhD, ; Britta RennerPhD,
}

\author{
Corresponding Author: \\ Laura Maria KönigBSc, MSc, PhD, \\ Phone: +497531885317 \\ Email: laura.koenig@uni-konstanz.de
}

\section{Abstract}

Background: Nutrition apps are a prototypical mobile health (mHealth) technology supporting healthy eating behavior that are seen as promising tools for health promotion by policy makers. Although nutrition apps are increasingly popular, wide-spread adoption is yet to be achieved. Hence, profound knowledge regarding factors motivating and hindering (long-term) nutrition app use is crucial for developing design guidelines aiming at supporting uptake and prolonged use of nutrition apps.

Objective: In this scoping review, we synthesized the literature on barriers to and facilitators for nutrition app use across disciplines including empirical qualitative and quantitative studies with current users, ex-users, and/or non-users of nutrition apps.

Methods: A systematic literature search including six databases as well as backward and forward citation search was conducted. Search strategy, inclusion and exclusion criteria, and the planned data extraction process were preregistered. All empirical qualitative and quantitative studies published in German or English were eligible for inclusion if they examined adults or adolescents (aged 13 to 18) who were either current users, ex-users and/ or non-users of nutrition apps. Based on qualitative content analysis, extracted individual barriers and facilitators were grouped into categories.

Results: Twenty-eight publications were identified as eligible. A framework with a three-level hierarchy was designed which grouped 326 individual barriers and facilitators into 21 sub-categories, twelve categories, and four clusters that focus on either the individual user (goals, goal attainment, goal abandonment, personal living conditions, lack of knowledge or skill, lack or loss of motivation, habit), different aspects of the app and the smartphone (features, usability of the app or food database, technical issues, data security, accuracy/trustworthiness, costs), positive and negative outcomes of nutrition app use, or interactions between the user and their social environment.

Conclusions: The resulting conceptual framework underlines a pronounced diversity of reasons for (not) using nutrition apps indicating that there is no "one-size-fits-all" approach for uptake and prolonged use of nutrition aps. Hence, tailoring nutrition apps to needs of specific user groups seems promising for increasing engagement.

(JMIR Preprints 12/05/2020:20037)

DOI: https://doi.org/10.2196/preprints.20037

\section{Preprint Settings}

1) Would you like to publish your submitted manuscript as preprint?

\section{$\checkmark$ Please make my preprint PDF available to anyone at any time (recommended).}

Please make my preprint PDF available only to logged-in users; I understand that my title and abstract will remain visible to all users.

Only make the preprint title and abstract visible.

No, I do not wish to publish my submitted manuscript as a preprint.

2) If accepted for publication in a JMIR journal, would you like the PDF to be visible to the public?

Yes, please make my accepted manuscript PDF available to anyone at any time (Recommended).

Yes, but please make my accepted manuscript PDF available only to logged-in users; I understand that the title and abstract will remain v

$\checkmark$ Yes, but only make the title and abstract visible (see Important note, above). I understand that if I later pay to participate in <a 


\section{Original Manuscript}




\title{
Barriers to and facilitators for using nutrition apps: a scoping review and conceptual framework
}

\author{
Laura M. König ${ }^{1 * \#}$, Christiane Attig²*, Thomas Franke ${ }^{3}$, Britta Renner ${ }^{1}$ \\ ${ }^{1}$ Department of Psychology, University of Konstanz \\ ${ }^{2}$ Department of Psychology, Chemnitz University of Technology \\ ${ }^{3}$ Institute for Multimedia and Interactive Systems, University of Lübeck \\ * both authors contributed equally
}

\section{Abstract}

Background: Nutrition apps are a prototypical mobile health (mHealth) technology supporting healthy eating behavior that are seen as promising tools for health promotion by policy makers. Although nutrition apps are increasingly popular, wide-spread adoption is yet to be achieved. Hence, profound knowledge regarding factors motivating and hindering (long-term) nutrition app use is crucial for developing design guidelines aiming at supporting uptake and prolonged use of nutrition apps.

Objective: In this scoping review, we synthesized the literature on barriers to and facilitators for nutrition app use across disciplines including empirical qualitative and quantitative studies with current users, ex-users, and/or non-users of nutrition apps.

Methods: A systematic literature search including six databases as well as backward and forward citation search was conducted. Search strategy, inclusion and exclusion criteria, and the planned data extraction process were preregistered. All empirical qualitative and quantitative studies published in German or English were eligible for inclusion if they examined adults or adolescents (aged 13 to 18) who were either current users, ex-users and/ or non-users of nutrition apps. Based on qualitative content analysis, extracted individual barriers and facilitators were grouped into categories.

Results: Twenty-eight publications were identified as eligible. A framework with a three-level hierarchy was designed which grouped 326 individual barriers and facilitators into 21 sub-categories, twelve categories, and four clusters that focus on either the individual user (goals, goal attainment, goal abandonment, personal living conditions, lack of knowledge or skill, lack or loss of motivation, habit), different aspects of the app and the smartphone (features, usability of the app or food database, technical issues, data security, accuracy/trustworthiness, costs), positive and negative outcomes of nutrition app use, or interactions between the user and their social environment.

Conclusions: The resulting conceptual framework underlines a pronounced diversity of reasons for (not) using nutrition apps indicating that there is no "one-size-fits-all” approach for uptake and prolonged use of nutrition aps. Hence, tailoring nutrition apps to needs of specific user groups seems promising for increasing engagement.

Keywords: nutrition apps, mobile health, mHealth, digital health, usage facilitators, usage barriers

${ }^{*}$ Corresponding author:

Dr. Laura M. König

University of Konstanz

Department of Psychology

P.O. box 47

78457 Konstanz

Phone: +49 7531885319 
Fax: +49 7531885226

Email: laura.koenig@uni-konstanz.de 


\section{Introduction}

Mobile digital health services for disease prevention or management (mHealth) [1] have become increasingly popular in recent years [2]. Currently, more than 300,000 mHealth apps are available in various app stores worldwide [3]. While mHealth services exist for a range of health behaviors and conditions including the management of chronic conditions such as hypertension (e.g., [4,5]) or mental health promotion [6,7], most freely available mHealth apps target lifestyle behaviors such as physical activity and eating behavior $[8,9]$. These mobile interventions have been shown to be effective for increasing physical activity [6,10], reducing sedentary behavior [11], and improving diet and diet-related health outcomes such as Body-Mass Index (BMI) [12]. Consequently, the widespread adoption of mobile phones - more than three billion people worldwide own a smartphone [13] - constitutes a promising opportunity to positively impact health behaviors. However, a large share of the general population does not yet use mHealth services [14-17]. Thus, the European Union aims at making web-based health promotion, including mHealth apps, more effective, user-friendly, and widely acceptable [18,19].

To boost mHealth service use by increasing acceptance and adoption and, ultimately, to target potential large-scale health effects, knowledge regarding why users decide for or against use is necessary. Moreover, examining the factors for sustained use opens the possibility to advance successful interaction with mHealth services. Research in the area of wearable health devices (e.g., fitness trackers, smartwatches) has already shown a large variety of reasons for device uptake, sustained used, and abandonment (e.g., [20-22]). Based on a systematic review, Simblett et al. [23] derived a comprehensive model of barriers to and facilitators of engagement with remote tracking technologies (e.g., health wearables, wireless monitoring devices). The authors identified health status, usability, convenience/ accessibility, perceived utility, and motivation both as key barriers and facilitators for use of remote mHealth technologies. Moreover, Attig and Franke [24] synthesized past research and identified a broad range of factors related specifically to fitness tracker abandonment, among these usability and measurement issues, demotivation, privacy concerns, disruption of the tracking routine, and successful habit formation. Thus, a range of reasons both related to the individual (e.g., health status, motivation, health-related goals) and technology (e.g., usability, accessibility, measurement inaccuracy) seem to impact the uptake and continued use of mHealth services for physical activity promotion.

Besides mHealth services for physical activity and exercise promotion, nutrition apps are one of the most frequently used mHealth services for promoting a healthy lifestyle $[8,9]$. However, 
mHealth services targeting these two behaviors differ in central aspects. For instance, nutrition apps usually require manual input of consumed foods to provide feedback, e.g., by having users enter foods and estimated serving sizes using a comprehensive food database. Fitness apps and wearables, on the other hand, allow for automatic data collection based on various sensor technologies (e.g., accelerometer, gyroscope). Hence, reliability of these two mHealth services refers to different data sources - the user vs. technology - and might thus be perceived differently by users. Furthermore, the difference in active vs. passive data collection might impact the motivation to use the services long-term, as tracking food intake manually might be more effortful compared to tracking physical activity automatically [15]. Finally, mHealth services for nutrition and physical activity differ in the type and temporal pattern of feedback provided. Regarding wearable fitness trackers and fitness apps, due to automatic and continuous collection of (in-)activity data, feedback can be provided continuously, also while an activity is taking place. This allows for adjustments to take place immediately. For nutrition apps, on the other hand, data is provided for distinct eating occasions. Hence, feedback can only refer to these distinct events. Moreover, feedback is often only available after the food was consumed or at least chosen. Thus, immediate adaption of the behavior might not be possible, which might impact how feedback is perceived and evaluated. It could therefore be hypothesized that barriers and facilitators for nutrition apps differ at least in part from barriers and facilitators for the use of fitness apps and wearables. Finally, these differences might also explain why fitness apps are generally more popular than nutrition apps [15].

Reasons specifically for nutrition app (non-)use were already explored in several studies (e.g., $[25,26])$. In a systematic review, Lyzwinski et al. [27] identified several features of text-messaging and app-based weight loss interventions that are related to uptake and continued use, including personalization, characteristics of messages, simplicity, entertainment and usability. Another review identified eight factors influencing user engagement with digital weight management interventions, including social support and the presence of certain features such as self-monitoring, prompts, and feedback [28]. Both reviews, however, focused on mobile interventions for weight loss and management, while nutrition apps can be used for a variety of goals, including self-monitoring [29], eating healthier [30], or even gaining weight [31], which again may reflect a variety of underlying motivations including health status (c.f., [23]) and specific needs and expectations. Furthermore, the methodological focus of the reviews was restricted: For instance, Lyzwinski et al. [27] included only qualitative studies. While qualitative research allows for a great richness of participants' responses, quantitative research usually comprises larger sample sizes and may allow for formal testing of theory, such as the Technology Acceptance Model [32]. Most studies included in Sharpe et al. [28], 
on the other hand, were randomized controlled trials which either tested the effect of the presence or absence of certain features (e.g., social network, tailored content) on engagement indices or evaluated features of one specific intervention, which might not be generalizable to all mobile eating interventions. It was thus deemed necessary to review the literature more broadly to reflect a wider range of app-based nutrition interventions, study designs and user perspectives.

The objective of the present review was to synthesize the literature on both barriers to and facilitators for the uptake and continued use of nutrition apps to provide a comprehensive overview of factors that hinder or promote use which can be used as starting points for nutrition app development and optimization. Studies with all possible user groups (non-users, users, ex-users, not specified) were included to allow for the identification of barriers and facilitators related to uptake, continued used as well as disengagement [15]. Even though some categories of previous reviews on mHealth for physical activity [23,24] may be transferred to nutrition apps (e.g., usability, goals, motivation), other categories might not fit their specific characteristics (e.g., discomfort issues as identified in Attig and Franke [24]). In addition, due to the broader scope than the reviews on weight loss and weight management apps [27,28], it could be expected that more and qualitatively different barriers and facilitators might be identified. Thus, due to this wide methodological scope and expected heterogeneous body of literature, a scoping review was conducted as it allows to summarize research findings and map key concepts without the need for a pre-determined coding scheme $[33,34]$.

\section{Methods}

A protocol for this scoping review was prepared and published on the Open Science Framework (https://osf.io/64d2h/) prior to completion of data extraction. Reporting is guided by the PRISMA-ScR [35] (see Online Supplementary Material).

\section{Data sources and search}

We searched the following databases through week 3 of May, 2019: Pubmed, Web of Science, PsychINFO, Psyndex, PsycArticles, and SPORTDiscus. For Pubmed, Web PsychInfo, Psyndex, PsycArticles, and SPORTDiscus a Boolean search term was used: ( " nutrition app*" OR "diet app*" OR "weight loss app*" OR "weight control app*" OR "weight management app*" OR "food journal" OR "health app*" OR "personal quantification" OR "quantified self" OR "personal informatics")) AND (adoption OR adherence OR abandonment OR attrition OR barriers OR motivation OR attitude OR *engagement OR 
"former user" OR ex-user OR "*continued use"). A slightly modified term was used for Web of Science due to differences in use of the asterisk (i.e., we had to add disengagement and "discontinued use"). No restriction was placed on publication date. Moreover, we conducted backward citation search by scanning the included studies for additional relevant references. We also conducted a forward citation search in Google Scholar using the included studies to complement the data search.

\section{Study selection}

\section{Inclusion and exclusion criteria}

Eligible studies examined factors hindering uptake or continued use (i.e., barriers) and/ or factors promoting update or continued use of nutrition apps (i.e., facilitators). To be eligible for inclusion, studies had to examine adults or adolescents (aged 13 to 18) in the following participant groups: current users, ex-users and/ or non-users of nutrition apps either for themselves or for their children. Studies with children and adolescents younger than 13 and health care providers using nutrition apps for patient support were excluded. Only empirical articles were included (i.e., literature reviews, meta-analyses, and conference abstracts were excluded). All study designs including qualitative or quantitative methodologies were eligible for inclusion. Studies had to investigate general nutrition app use (i.e., studies evaluating particular apps were excluded). Moreover, studies focusing more broadly on the use of health apps were included as long as they specifically stated that nutrition apps were included. Studies were only eligible if the examined apps included an assessment of diet such as logging consumed foods (e.g., studies evaluating sole weight logging apps were excluded). Further, we included only English and German articles. Finally, two studies known to authors from other sources were also included.

\section{Screening}

Authors LK and CA independently reviewed titles and abstracts, and, subsequently, full-texts according to the inclusion and exclusion criteria. Conflicts were resolved through discussion until consensus was achieved.

\section{Data extraction and collation}

Authors LK and CA reviewed each full-text article independently and extracted data on facilitators and barriers to nutrition app use (i.e., direct quotes from articles). Subsequently, all authors categorized facilitators and barriers manually according to principles of qualitative content analysis (i.e., inductive category development [36]). Differences in abstraction were resolved by discussion until consensus regarding category logic (i.e., no overlaps of contents across categories) 
was achieved. The inducted categories were first defined, then compared and harmonized with the extracted quotes. The final category system including the underlying quotes was documented into an Excel spreadsheet and can be found in the Online Supplementary Material. Categories may contain both barriers and facilitators as several aspects of nutrition apps and their use were not universally perceived to be positive or negative (e.g., app usability can both be a facilitator and barrier depending on the individual user's perception). In a final step, the categories were grouped into higher-order clusters based on their origin or source as they might provide further insight into starting points for improvement.

In addition, author LK reviewed each full-text article and extracted the following study characteristics into Excel spreadsheets: app user group (all, users, or users and ex-users), sample size, age, gender, specificities of the study sample (general population or patients), study design (qualitative, quantitative, mixed), study location and type of app (nutrition vs. health app; see Table 1 and Online Supplementary Material Table 3).

Table 1. Overview of included studies.

\begin{tabular}{llllll}
\hline Publication & N & $\begin{array}{l}\text { Target } \\
\text { group }\end{array}$ & $\begin{array}{l}\text { App } \\
\text { user } \\
\text { group }\end{array}$ & $\begin{array}{l}\text { Study } \\
\text { design }\end{array}$ & $\begin{array}{l}\text { Type of Location } \\
\text { apps }\end{array}$
\end{tabular}

\begin{tabular}{|c|c|c|c|c|c|c|}
\hline Aljuraiban [37] & 1191 & adults & all & quantitative & $\begin{array}{l}\text { health } \\
\text { apps }\end{array}$ & Saudi Arabia \\
\hline $\begin{array}{l}\text { Anderson et al. } \\
\text { [38] }\end{array}$ & 22 & adults & user & qualitative & $\begin{array}{l}\text { health } \\
\text { apps }\end{array}$ & Australia \\
\hline $\begin{array}{l}\text { Bhuyan et al. } \\
\text { [39] }\end{array}$ & 3677 & adults & all & quantitative & $\begin{array}{l}\text { health } \\
\text { apps }\end{array}$ & US \\
\hline Chan et al. [40] & 20 & $\begin{array}{l}\text { adolescents } \\
(13-18 \\
\text { years of age) }\end{array}$ & all & qualitative & $\begin{array}{l}\text { health } \\
\text { apps }\end{array}$ & US \\
\hline Choe et al. [41] & 52 & adults & user & qualitative & $\begin{array}{l}\text { health } \\
\text { apps }\end{array}$ & $\begin{array}{l}\text { international } \\
\text { (US, UK, } \\
\text { Singapore) }\end{array}$ \\
\hline $\begin{array}{l}\text { Cordeiro et al. } \\
\text { [26], Study } 1\end{array}$ & 141 & adults & $\begin{array}{l}\text { user, } \\
\text { ex- } \\
\text { user }\end{array}$ & quantified & $\begin{array}{l}\text { nutritio } \\
\text { n apps }\end{array}$ & N/A \\
\hline Cordeiro & 5526 posts & N/A & user, & quantified & nutritio & N/A \\
\hline
\end{tabular}


[26], Study 2

Cordeiro et al. 257 adults [25]

Dennison et al. 19 [42]

Eikey and Reddy 16 [31]

Flaherty et al. 12 [43]

Gowin et al. [44] 27

Haithcox-Dennis 1487

et al. [45]

Jones et al. [30] 195

Krebs

and 1604

Duncan [29]

Kwon et al. [46] 391

adults

adults

Lieffers et al. 24

[47]

Murnane et al. $\mathrm{M}=1839$ adults [48]

responses

per

question

Oh and Lee [49] N/A

N/A

adults ex-

n apps

user

user, quantified nutritio N/A

ex- $\quad n$ apps

user

all qualitative health UK

apps

user, qualitative nutritio US

ex- $\quad n$ apps

user

user qualitative nutritio Ireland

n apps

user qualitative health US apps

all quantitative health US apps

all quantitative health US apps

all quantitative health US apps

all quantitative health US apps

user, qualitative nutritio Canada

ex- n apps

user

all quantitative health US

apps

user, quantified health N/A

ex- apps

user

all qualitative health US apps 


$\begin{aligned} & \text { Sarcona et al. } 401 \quad \text { adults all quantitative health US } \\ & \text { [51] }\end{aligned}$
apps

\begin{tabular}{|c|c|c|c|c|c|c|}
\hline Solbrig et al. [52] & 24 & adults & all & qualitative & $\begin{array}{l}\text { nutritio } \\
\text { n apps }\end{array}$ & UK \\
\hline Tang et al. [53] & 19 & adults & $\begin{array}{l}\text { user, } \\
\text { ex- } \\
\text { user }\end{array}$ & qualitative & $\begin{array}{l}\text { nutritio } \\
\text { n apps }\end{array}$ & UK \\
\hline $\begin{array}{l}\text { Wang et al. [10], } \\
\text { Study } 1\end{array}$ & 23 & adults & all & qualitative & $\begin{array}{l}\text { health } \\
\text { apps }\end{array}$ & Norway \\
\hline $\begin{array}{l}\text { Wang et al. [10], } \\
\text { Study } 2\end{array}$ & 500 & adults & all & quantitative & $\begin{array}{l}\text { health } \\
\text { apps }\end{array}$ & Norway \\
\hline $\begin{array}{l}\text { Warnick et al. } \\
\text { [54] }\end{array}$ & 18 & adults & all & qualitative & $\begin{array}{l}\text { health } \\
\text { apps }\end{array}$ & US \\
\hline West et al. [55] & 217 & adults & $\begin{array}{l}\text { user, } \\
\text { ex- } \\
\text { user }\end{array}$ & quantitative & $\begin{array}{l}\text { nutritio } \\
\text { n apps }\end{array}$ & US \\
\hline $\begin{array}{l}\text { Woldeyohannes } \\
\text { and Ngwenyama }\end{array}$ & 11 & adults & user & qualitative & $\begin{array}{l}\text { health } \\
\text { apps }\end{array}$ & Canada \\
\hline
\end{tabular}

[56]

$\begin{array}{lllllll}\text { Yuan et al. [57] } & 317 & \text { adults } & \begin{array}{l}\text { user, } \\ \text { ex- } \\ \text { user }\end{array} & \text { quantitative } & \begin{array}{l}\text { health } \\ \text { apps }\end{array} & \text { US } \\ \text { Zhou et al. [58] } & 117 & \text { adults } & \text { all } & \text { mixed } & \begin{array}{l}\text { health } \\ \text { apps }\end{array} & \text { US }\end{array}$

\section{Results}

\section{Literature search}

A total of 2654 individual records were screened. After 2480 were excluded when screening titles and abstracts, 174 full texts were screened for eligibility. Subsequently, 28 publications containing 30 studies were included (see Figure 1 for a flow diagram).

\section{Characteristics of the included studies}

The $N=28$ publications were all published between 2012 and 2019. Two publications contained two studies while the remaining 26 publications each reported results from one study. 
Almost all samples comprised adults from the general population, while one publication focused on adolescents and one publication focused on women with an eating disorder. Nine publications focused specifically on barriers and facilitators of nutrition app use (e.g., diet tracking feature in MyFitnessPal, LoseIt!), while 19 publications investigated barriers to health apps in general, but explicitly included nutrition apps such as calorie counters. Fifteen of 28 included publications did not focus on a specific user subgroup, while five publications focused on users and eight studies included both users and ex-users. For the majority of publications, data were collected in the US (15/28), followed by the UK (3/28) and Canada (2/28). One publication presented data from multiple countries. See Table 1 for an overview of included studies.

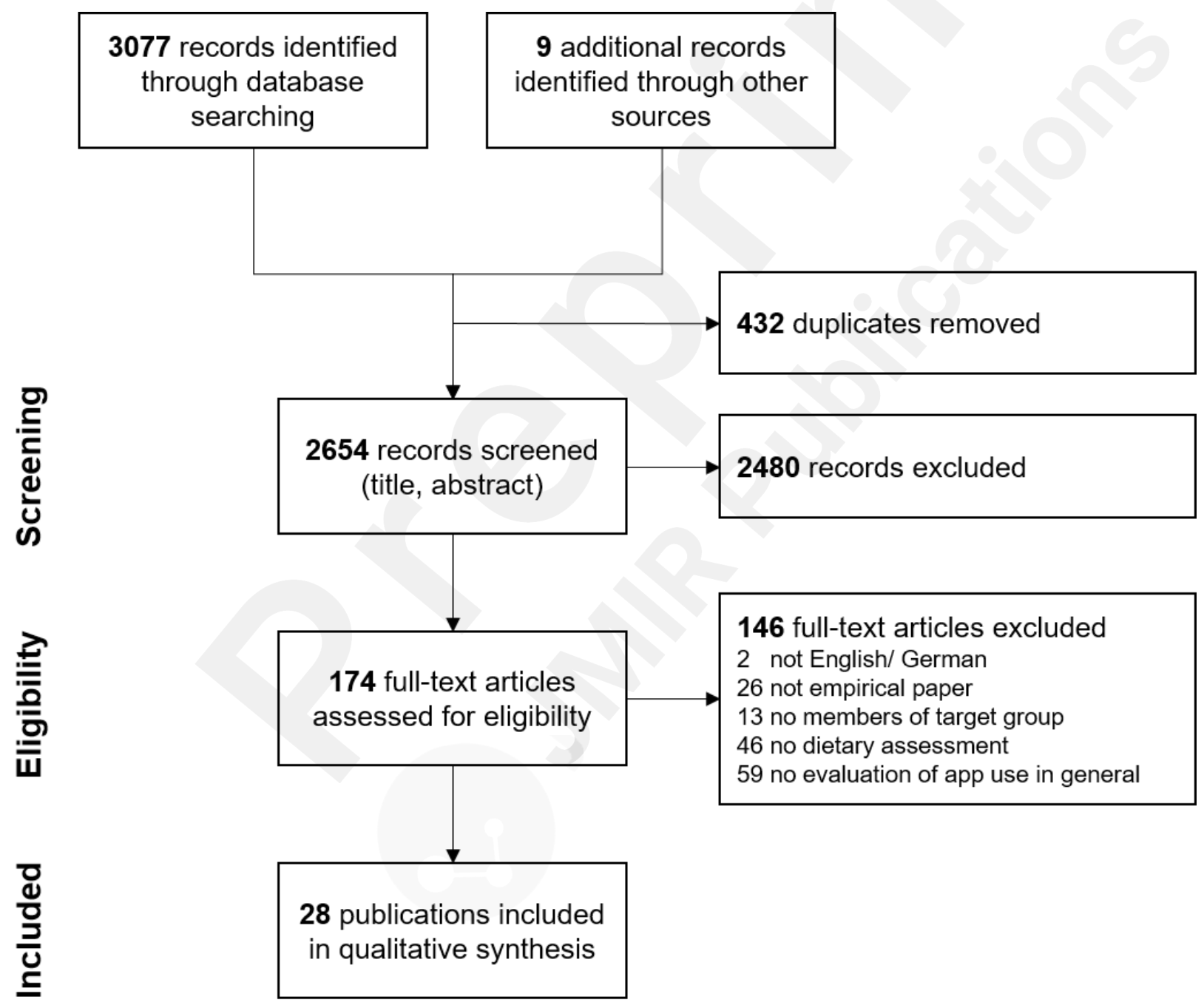

Figure 1. Flow diagram of article selection.

\section{Barriers to and facilitators for nutrition app use}

A total of 326 barriers to and facilitators for nutrition app (non-)use were extracted from the publications. The number of extracted barriers and facilitators varied greatly between publications 
$(M=11.61, S D=8.55$; range 2-39)

While grouping barriers and facilitators into categories, a three-level hierarchical framework emerged (see Figure 2 for a schematic overview of the framework). First, barriers and facilitators were grouped into 21 sub-categories. Second, several categories were clustered (e.g., lack of interest and declining motivation were both related to motivation, thus grouped together), resulting in 12 categories (C1-C12; note that C4 and C8-C11 do not consist of sub-categories). Third, the resulting categories were grouped into higher-order clusters that focus on either (1) the individual (C1-C4), (2) the app and the smartphone (C5-C9), (3) intended and non-intended outcomes of nutrition app use (C10-C11), or (4) the social environment (C12).

Barriers and facilitators within the individual were related to [C1] goal setting and goal striving (i.e., [C1a] the type of goal, [C1b] goal attainment, and [C1c] goal abandonment), [C2] motivation (i.e., [C2a] lack of interest and [C2b] declining motivation), [C3] tracking routines (i.e., [C3a] fit of the app to daily routines and circumstances as well as [C3b] tracking habit), and [C4] lack of knowledge or skill. Barriers and facilitators related to the app and the smartphone were [C5] app features (i.e., [C5a] presence of features as well as [C5b] personalization), [C6] usability of the app ([C6a]) and the food tracking feature ([C6b]), [C7] trustworthiness regarding data accuracy ([C7a]) as well as data security and privacy ([C7b]), [C8] technical issues, and [C9] financial costs of the app. In addition, barriers and facilitators were identified that stem from using the app, which include intended and non-intended [C10] positive outcomes and effectiveness as well as [C11] negative outcomes. Finally, barriers and facilitators regarding [C12] the social environment (i.e., [C12a] recommendation to use and [C12b] social interactions) were identified.

There was a substantial variation in the number of sub-categories targeted by the different publications ( $M=6.75, S D=3.81$; range 1-15 of 21 sub-categories) and accordingly, the frequency of the sub-categories being mentioned across publications varied substantially $(M=8.90, S D=4.59$; range 1-19 of 28 publications; see Table 2 in the Online Supplementary Material for details). 


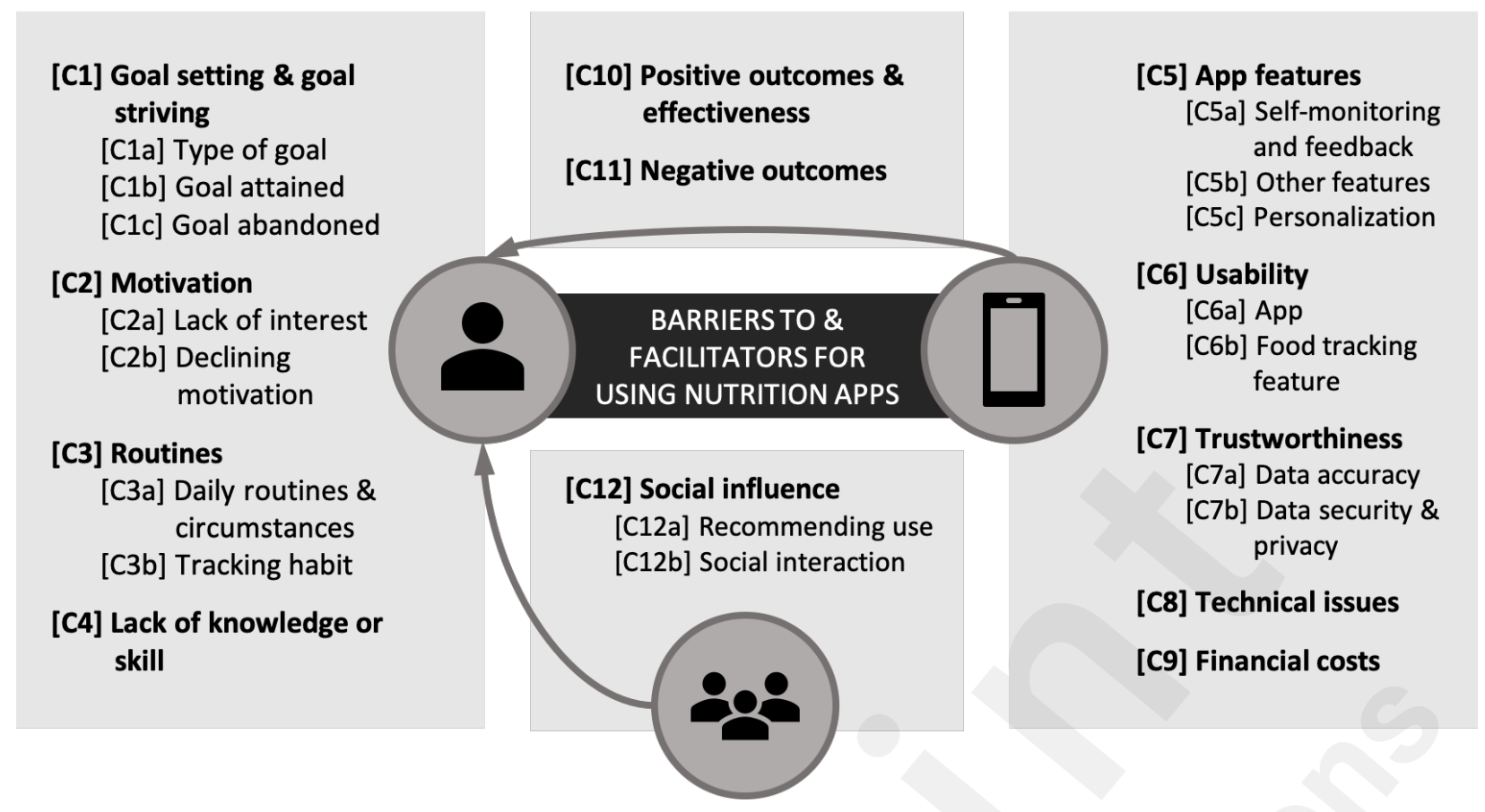

Figure 2. Framework comprising 12 categories [C1]-[C12] and respective sub-categories of barriers and facilitators identified in the individual studies. Higher-order clusters refer to barriers and facilitators originating from the individual, the app and the smartphone, nutrition app use, and the social environment.

\section{[C1] Goal setting and goal striving [C1a] Types of goals}

In 15 publications, the type of goal was mentioned as a facilitator for nutrition app use. Nutrition-related goals such as food tracking [29,31,47,51] or diet improvement $[30,43,44,55]$ were frequently named. Other participants stated to use apps for weight management [51]: most of them aimed to lose weight [31,44,48,52,53] whereas some participants also used an app to eat more [31].

Moreover, some participants had a number of goals related to health behaviors beyond nutrition that they felt they needed assistance with and therefore used the app for $[39,41,44,45,47,57]$. Some participants intended to improve their health [49], to be more mindful or to find balance [41]. Others wanted to adopt a new or maintain an existing behavior [44]. Again others needed assistance with medical or health-related decision-making [39,41], aimed to increase their knowledge in order to answer specific questions, to find triggers [41], or to be able to ask their physician more specific questions or to ask for a second opinion [39]. Finally, some participants stated to use apps to cure or manage a condition or to execute a treatment plan [41].

Finally, Choe et al. [41] identified further goals related to other aspects of life and new life experiences, such as maximizing work performance [41]. These results highlight that nutrition apps may be used for a great variety of different goals ranging from highly specific nutrition-related goals 
to very general health-related goals to improvements in other aspects of life.

\section{[C1b, C1c] Goal attained or abandoned}

Two reasons for disengagement with an app that were related to goals could be identified. On the one hand, app use may no longer be necessary if the goal was reached or a desired habit was formed $[25,47,48,50]$, which was identified in four publications. On the other hand, one publication highlighted that goals may be abandoned and the app along with it [48].

\section{[C2] Motivation}

\section{[C2a] Lack of interest}

In four publications, nonusers expressed a general lack of interest in using health apps including nutrition apps because they felt that they do not need to use one $[29,45]$. Notably, reasons for this included the availability of other tools such as paper and pencil diaries [50], or competing interests such as preferring to use their smartphones for other apps such as social media [40].

\section{[C2b] Declining motivation}

Furthermore, in seven publications, some (ex-)users reported that their motivation to use apps may decline over time [25,29,37,43]. Potential reasons for this might be limited progression [47] or boredom [40,42], e.g., because the app only provides a limited range of functionalities [56].

\section{[C3] Routines}

\section{[C3a] Daily routines and circumstances}

Five of the reviewed publications highlighted that the fit between the user's daily routines or current living situation and app might impact uptake and adherence [47]. For instance, participants in one study [48] reported that they discontinued using an app because they were not able to use it in certain living environments. Others criticized that using an app interfered with their daily activities and social life [51]. Dennison et al. [42] highlighted that apps were most likely to be used if they were well integrated into users' usual smartphone use patterns. Finally, in a study with adolescents, participants reported that apps were not targeted towards their age group, but adults [40]. Thus, according to the reviewed studies, nutrition apps need to fit to the user's daily routines as well as their general life circumstances.

\section{[C3b] Tracking habit}

In four publications, a (lack of) tracking habit was identified as an influencing factor for nutrition app use. Some participants reported that they stopped using an app because they forgot to use it in daily life [25,26,42]. Similarly, Yuan et al. [57] showed that apps were less likely to be 
abandoned when a habit of using the app was formed.

\section{[C4] Lack of knowledge or skill}

Four publications highlighted the importance of knowledge and skill especially for nutrition app uptake. Some of the nonusers surveyed in the reviewed studies reported not to be aware that health and nutrition apps existed [45,50]. Others may be aware of this type of apps, but are unsure which one to use, lack awareness of specific functionalities and capabilities, or do not know how to use them properly $[40,47,50]$.

\section{[C5] App features}

\section{[C5a] Self-monitoring and feedback}

In the 12 publications which mentioned self-monitoring and feedback features, inclusion of these features [43,56] such as food diaries or food scanners [53] was often seen favorably. In three studies, participants explicitly expressed their satisfaction with comprehensive food databases to record food intake $[47,53,56]$ and in one further study, participants criticized databases that were too limited [26]. Specifically, Lieffers et al. [47] noted that participants preferred larger over smaller food databases as these saved time and were convenient. Furthermore, app users appreciated the opportunity to view a history of tracked data without visiting a medical doctor, or having the opportunity to send data to health professionals remotely [38]. Similarly, users in Aljuraiban [37] were more likely to discontinue use if monitoring by a specialist was not offered.

Regarding feedback features there were disagreements on how messages should be designed [47]: Some participants stated that they did not like to count calories [52] or to restrain themselves based on feedback from the app [42]. Regarding information presentation, participants suggested using various media formats (e.g., video, audio) [40] and visualizations [49]. Also, they were keen that a high level of detail was preserved in the feedback [42]. Finally, participants preferred to access information on the go [53]. To sum, users of nutrition apps might generally expect extensive and engaging food tracking features to be included in nutrition apps, while they might differ in their preferences for content and design of the feedback.

\section{[C5b] Other features}

The impact of the presence or absence of other features were mentioned in 13 publications. Inclusion of features supporting self-regulation such as rewards $[40,41,49,50,53]$ and goal-setting $[48,50]$ was appreciated. Moreover, participants generally valued the provision of nutrition knowledge that they did not already have or might not be able to access otherwise $[43,50,52]$. 
Other features, however, were not universally appreciated, such as prompts and reminders. While some participants stated that they were helpful [42,43,50,53], others reported to be annoyed by too frequent notifications $[42,47,56,58]$., e.g., to update the app regularly and thus ensure its functionality [38]. Similarly, while some reported to like the idea of gamified elements within an app [43,50], others perceived them to be demotivating [43].

Finally, the absence of desired or helpful features was mentioned by participants in several studies $[47,49,56]$, which might lead to abandonment of the app [48]. For instance, in two studies, participants highlighted the need for integration with other apps, e.g., to synchronize calorie consumption and expenditure $[47,49]$. Poor integration with other apps might lead to disappointment with an app and to subsequent disengagement [47]. On the other hand, integration of unnecessary features such as location tracking was criticized in Dennison et al. [42]. However, based on the included quote, one can only speculate whether these features will actually lead to abandonment of an app or simply be ignored. Including desired features in nutrition apps might be an important facilitator of nutrition app uptake and use. However, inconsistent opinions regarding many app features were identified, which highlights that the inclusion of app features might need to be tailored to individual needs.

\section{[C5c] Personalization}

Independent of the specific feature, participants in eleven publications underlined that features should be customizable and tailored to individual needs and goals [37,38,43,48,50,52,53]. For instance, participants in Dennison et al. [42] valued the opportunity to set customized reminders and having choice in message content and tone, and participants in Chan et al. [40] stated that they would like to receive personalized information and coaching through an app. While some participants stated to like apps that provided a pre-specified list of goals to choose from $[47,48]$, some participants stated that they would prefer to set individual goals as they were unsatisfied with the provided ones [43,47]. In addition, tailoring to ethnic preferences was valued [56]. By allowing users to customize the app, personal autonomy, freedom of choice, and a feeling of congruency is preserved, which might positively influence the likelihood of continued use [43]. Thus, as needs and expectations regarding the design and content of certain app features may vary between users, customization or variation of both features and app content in general may thus be helpful to prevent abandonment. 


\section{[C6] Usability}

\section{[C6a] Usability of the app}

In 19 publications, usability of the app was seen as an important precursor of nutrition app uptake and continued use, which makes it the category that was identified most frequently. Participants criticized that apps were too confusing, complex and stressful, both when setting them up [42,44] and when using them [26,29,37,40,42,44,47,50,52,56], e.g., because of lengthy instructions [42]. For instance, participants in Gowin et al. [44] stated that they would not download apps that required lengthy instructions. Accordingly, easy and simple tracking procedures were valued by users [41] and perceived ease of setup and use increased the likelihood for adoption and continued use $[30,38,43,45,47,53,55]$.

In a similar vein, participants stated to dislike apps that were time-consuming to use [42,43,54], which might lead to increased levels of stress [10]. Lack of time might thus be considered an important barrier to using nutrition apps [50].

Finally, also interface design aspects were mentioned in a few studies. Specifically, studies highlighted that an attractive design may increase the likelihood for an app to be used [40,53]. Ease of use, time required for use and attractiveness thus may impact whether and how frequently a nutrition app is used.

\section{[C6b] Usability of the food tracking feature}

Food tracking features are an integral part of nutrition apps. Their usability was criticized in seven of the reviewed studies. Usability of the food tracking feature might therefore constitute an important influence on the likelihood for (continued) use of a nutrition app. Especially the ease of use of food databases was seen critically. Many users of nutrition apps reported to have experienced difficulties when entering data [25,56]. In particular, they reported difficulties identifying the correct foods because of too many options [47], finding the correct foods because they were missing from the database [26] or entering the correct foods and portion sizes [47]. Providing detailed entries and entering homemade food or meals consumed in a restaurant were seen to be especially challenging [26]. Participants might even cease to enter their foods, for instance when consuming a variety of foods over a longer time-span [26].

Furthermore, the time needed for food journaling was seen crucial by participants, as they stated not to want to spend much time on entering their foods into the app $[26,47,49]$. Entering foods being too time consuming was named as an important reason for not using or ceasing to use nutrition apps [25,29]. Participants would therefore prefer automated tracking functions [41] or food scanners 
[53]. However, Lieffers et al. [47] noted that several participants preferred using an app-base food database to other non-digital methods such as paper diaries because apps were perceived as more convenient and less time-consuming. Difficulty and time thus seem to be especially important factors that should be taken into account when designing food databases in nutrition apps.

\section{[C7] Trustworthiness}

\section{[C7a] Data accuracy}

A (lack of) concern regarding data accuracy was identified in seven publications. While some participants see nutrition apps as a trusted ally that supports them to achieve their goals [43], other participants were concerned regarding the accuracy and trustworthiness of information presented in the app [49]. Some participants expressed concern regarding human error when tracking food intake, as tracking tools might allow to deliberately adjust entries which may lead to inaccurate records [42]. Other participants stated to be concerned about tracking errors within the database or the app itself [26,42], e.g., missing or duplicate food entries or incorrect caloric information [47], or to be unsure whether the provided information, e.g., in discussion boards, was accurate and could be trusted [42,53]. Moreover, participants criticized that apps may be misguiding regarding the predicted accuracy of provided information [56]. Worries regarding the app's accuracy and trustworthiness might therefore stem from two sources: the individual using the app or features and underlying information sources such as food databases.

\section{[C7b] Data security and privacy}

A number of data security concerns were mentioned by participants in ten of the reviewed publications (e.g., [42,56]). Some participants were unsure what the smartphone might do with the data without their awareness [42]. They expressed worry that their potentially sensitive data would be made available to third parties like health insurers [38] or companies, e.g., to tailor advertisements to them [42], although they would not consent for the data to be shared [50]. Especially location sensing was seen critically. Participants expressed concern because it might be a risk to personal safety, e.g., when GPS data was accessed by burglars [42,49]. Accordingly, participants were unsure whether they could trust the apps and their developers [29,48], and apps provided by health experts like medical doctors were seen as more trustworthy and persuasive [42]. Other participants, however, stated to be unconcerned because they thought their data would not be of interest to third parties [42].

Zhou et al. [58] specifically focused on data security concerns and barriers and facilitators to the use of mobile health apps including nutrition apps. Concerns were raised because of storing unencrypted personal data on the smartphone, sending data to remote servers without permission, 
and a general lack of privacy statements in many apps.

Especially in apps with in-app community features or connections to social media, anonymity was important to app users [43]. There were different opinions, however, whether participants approved of sharing data with a community. While some current users were favorable of sharing their data online [49], Krebs and Duncan [29] reported that apps sharing data with friends was a frequently named reason for abandoning an app. Sharing might be disliked especially if participants feel that they cannot control which and how much information is shared [42]. Besides ensuring that data is stored safely and privacy is respected it may thus also be important to make data protection efforts transparent to potential users.

\section{[C8] Technical issues}

An important prerequisite for being able to use nutrition apps is compatibility of the app with one's smartphone [48]. However, even if the smartphone fulfils an app’s basic system requirements, a number of technical issues might decrease the likelihood of app adoption and continued use. Accordingly, technical issues were identified as a potential barrier in eight publications. Apps may slow down the smartphone [58] and thus may impair the use of other apps or phone functions [42] or lead to crashes [47]. Furthermore, excessive battery drain [42,48,50], use of memory and/ or space [42,50] and use of mobile data [29] were criticized in several studies. Moreover, technical issues within the app such as app dysfunctions [56] and inconvenient data transfer [49] may lead to frustration and subsequent disengagement. Finally, users might disengage from using an app because of concerns related to radiation from the smartphone [50]. App developers therefore might need to find a balance between using advanced but resource intensive features and ensuring compatibility with many different smartphones that vary in age and technical features.

\section{[C9] Financial costs}

Financial costs of nutrition apps were identified in eight publications. While one participant in Lieffers et al. [47] stated that they were more motivated to use the app because they paid for it, participants across several studies reported that the price and costs of in-app purchases were factors hindering app use [29,45,50], especially since there are free apps available [44,58]. Some participants, however, said that they might be willing to pay some money for the app if it was good and provided a good price value [44,57]. Furthermore, some participants criticized hidden costs (e.g., for enabling additional app features) [37], which may lead to disengagement [29]. Thus, financial costs might need to be low and made transparent to promote nutrition app use. 


\section{[C10] Positive outcomes and effectiveness}

In 16 publications, participants reported a number of positive outcomes of nutrition app use that were related to cognitions, emotions, and behavior and health, which tie in to perceived effectiveness of the app to promote healthy behaviors. Regarding cognitive outcomes, using an app may increase users' awareness and motivation for healthy eating [10,30,38,44,50,53,55] and improve their self-management skills [38]. Accordingly, nutrition apps are perceived to be informative and to promote nutrition knowledge [10,52]. Tracking may also provide participants with a sense of accountability and the ability to track progress over time [48] and improve their self-efficacy [43]. Regarding emotional outcomes, participants reported that using an app made them feel good about themselves [10] and their bodies [51]. Furthermore, apps may provide encouragement and support [44,52]. Moreover, participants in three studies reported that using the app was fun and enjoyable [55,57] which may positively influence engagement [49]. Finally, several users reported beneficial effects of use on their health [30,48,51]. Accordingly, nutrition apps are seen as potentially useful [46,55] as merely viewing tracked behavioral data may facilitate behavior change [42]. However, not all surveyed participants agreed with this notion and perceived nutrition apps not to be effective $[42,45]$.

\section{[C11] Negative outcomes}

A number of potential adverse consequences of app use on cognitions, emotions, and behavior and health were reported in twelve of the reviewed studies (e.g., [50,54]). First, studies reported an array of negative influences on cognitions: Participants reported obsession with food or calorie counting [26,47,51,52] and to be overly engaged with one’s states and behaviors [49].

Second, negative emotional consequences were named. Negative app-generated information including feedback based on tracking of food intake and messages sent by the app might evoke negative emotional reactions including disappointment, guilt, and anxiety [26,47,51,53,56], especially if users fall short of reaching a pre-determined goal [42]. Moreover, apps may also make users feel neurotic about their body image [51].

Third, using nutrition apps might also have negative impact on behavior and subsequently health. Some participants expressed worry that eating potentially unhealthy, but easy to log foods like ready meals would be rewarded and thus encouraged their consumption [25,26]. Other participants expressed concern that feedback on calorie consumption might backfire, e.g., when caloric intake and expenditure are tracked in combination and burned calories may be seen as a permission to eat more [47]. Finally, some participants stated to be concerned that apps promoting 
extreme calorie restriction might even lead to potential harm [56] including inducing or exacerbating an eating disorder [31]. Nutrition app use might therefore be promoted if expected or experienced negative consequences are attenuated.

\section{[C12] Social influence}

\section{[C12a] Recommending use}

Social influence might promote app uptake, as was highlighted in nine publications (e.g., $[40,46,56])$. For instance, in four of the reviewed publications, participants reported to have learned about apps from family members or friends $[44,45,48,50]$. Others said they learned about health apps from a health or fitness professional or from their employer [29,48]. Still others stated to have chosen the app based on recommendations and positive reviews in app stores, social media or TV $[45,46,48]$.

\section{[C12b] Social interactions}

Similarly, social interactions in the app or related to the app might influence nutrition app use, as was highlighted in eleven publications. For instance, participants valued competitions [50,56] and support functions [53]. Furthermore, Wang et al. [10] reported that sharing data over the internet might sustain motivation in users. When friends stop using the app, participants reported that it was more likely that they would stop using the app as well [26]. However, some participants also reported that learning about others' success or competing against other app users might be demotivating $[26,47,50]$.

On the other hand, perceived undesirability and stigmatization of using apps might hinder uptake and continued use [26,40,42] because it may be embarrassing [42]. Some participants stated, for instance, that they did not feel comfortable using an app in front of others [25,47] or did not even want other people to know that they were using an app because it might imply that they have a certain disease [58]. Thus, social influence might both promote and hinder nutrition app uptake and use, depending on whether attitudes towards nutrition apps in the social environment are positive or negative.

\section{Discussion}

Nutrition apps are less popular than fitness apps [15] although they might have comparably beneficial effects on health [11,12]. To better understand differences and dynamics in use, the present scoping review provides a hierarchical framework of barriers and facilitators for nutrition app use. The presented framework consists of 21 sub-categories, 12 categories, and 4 higher-order clusters and describes how technology, the individual, outcomes of their interaction, and the social 
environment may impact nutrition app uptake and continued use. The largest of the four clusters were barriers and facilitators related to technology (i.e., apps or smartphones), which consists of five categories and nine sub-categories. The framework moreover highlights that also characteristics of the (potential) user, the interplay between user and technology and the social environment impact whether a nutrition app is used. For instance, it underlines the importance of tailoring the app content to the user's goals, expectations and needs. As Villinger et al. [12] pointed out, nutrition apps mainly employ four categories of Behavior Change Techniques (BCTs; [59,60]) that primarily address constructs of deliberate behavioral control, such as goal setting, self-monitoring, and feedback (see also [61] for an analysis of commercial apps). However, by developing apps that address a more intuitive decision-making style, the number of nutrition app users might be increased [15].

Interestingly, previous research has put an even stronger emphasis on barriers and facilitators related to technology. For instance, most of the barriers and facilitators for nutrition app use identified in Sharpe et al. [28] were app- or smartphone-related. This might be explained by different foci of the reviews. Nine of the eleven included studies in Sharpe et al. [28] were evaluations of randomized controlled trials or ongoing weight management programs. Researchers might thus have been especially interested in usability evaluations of specific program features that support long-term engagement with the tested intervention. Including surveys of general population samples that are not restricted to participation in an intervention study yielded a greater variety of barriers and facilitators because some of these factors might not play a role for study participants. For example, lack of knowledge or skill might not emerge as a barrier when evaluating a digital weight management program because participants often attend a training session before the start of the study (e.g., [62]). Similarly, technical issues might not be as frequent as participants might be pre-selected based on the type of smartphone they use (e.g., [63]), or they might receive a smartphone from the study team to ensure compatibility (e.g., [64], Study 3). Also data security concerns might not be of great importance when taking part in a study at a university as researchers might be seen as more trustworthy [42]. Finally, financial costs might not play a role because using an app as part of a study is usually free. Thus, by explicitly including studies independent of the use of specific nutrition apps, the present review was able to generate a comprehensive list of barriers and facilitators that play a role when deciding whether to use (or continue to use) a nutrition app in daily life.

Previous reviews also often focused on specific user groups such as current users of nutrition apps [28] or remote tracking technology [23] or on ex-users of wearables [24], and thus lack the 
perspective of potential users who did not yet think about using mHealth technology for health promotion or decided against its use (for a discussion, see also [15]). The present review, on the other hand, included literature on nutrition apps in general and included current and ex-users of nutrition apps as well as nutrition app non-users and thus generated an extensive list of barriers and facilitators. Consequently, several sub-categories were predominantly or even exclusively identified in publications which included ex-users or non-users in addition to current nutrition app users. For instance, the category [C4] "lack of knowledge or skill” was not mentioned in publications that exclusively focused on current users, and the sub-category [C2a] "lack of interest" was only identified in publications which also included non-users. However, one could argue that these barriers are especially important to address in order to facilitate contemplating the use of nutrition apps, which is an important first step in the nutrition app adoption process [15, 65]. Similarly, the category [C3] "routines” with its sub-categories [C3a] “daily routines and circumstances” and [C3b] "tracking habit" were not identified in studies which focus exclusively on current users, presumably because current users have already established a tracking habit and are using an app that fits their daily routines. However, forming a habit of using a nutrition app might be an important prerequisite for prolonged nutrition app use (see [66] for a discussion of the importance of habits for behavior change) and therefore should be taken into account when developing nutrition apps.

Despite the differences in target populations and technologies, there is substantial overlap between barriers and facilitators to different mHealth technologies identified in the present review and previous reviews $[23,24,27,28]$. For instance, reviews on both nutrition apps and wearables identified facilitators and barriers related to data security and privacy, app features, or technical issues. Thus, many of the identified barriers and facilitators could be generalized across mHealth technologies, and the presented framework may provide insights for designing mHealth technologies more broadly.

\section{Implications for the development of nutrition apps}

The framework developed in this review highlights several starting points for the improvement of nutrition apps. For instance, usability was the most frequently identified barrier in the present review and was also identified in previous reviews [23,24,27,28]. Importantly, usability issues were identified both concerning the app in general (e.g., navigation through the app) and more specifically concerning the food tracking feature and the underlying food database. The latter constitute core features of many nutrition apps [67,68]. Previous research has shown that usability issues related to tracking of food intake might impact willingness to record eating events [69]. 
Therefore, deficient usability of the food database might also indirectly impact other categories of barriers and facilitators identified in this review such as accuracy and trustworthiness if fewer meals are recorded. Subsequently, this may also impact features that rely on accurate data, such as feedback. Usability of nutrition apps and especially food tracking features should therefore be a major concern for app developers. User burden can, for example, be reduced by using simpler input mechanisms, e.g., indicating portion sizes using common household items [70], or photo-based food recording $[67,71]$.

The presence of certain app features including self-monitoring and feedback features can be seen as a facilitator for continued nutrition app use (see also [28]). As Lyzwinski et al. [27] pointed out, the inclusion of BCTs is often valued by nutrition app users. It could thus be concluded that the inclusion of certain BCTs might not only increase effectiveness of interventions [72,73] but also engagement [74]. Moreover, both the present review and previous reviews highlighted that users appreciated opportunities for personalization of the app [23,27,28], which is also related to effectiveness in the literature $[61,75]$. It can therefore be recommended to include features such as feedback, goal setting, and prompting, and to allow for customization, e.g., by allowing users to set customized reminders, to increase engagement.

Finally, anticipated or experienced positive and negative consequences, including the (lack of) effectivity, were among the most frequently identified reasons for nutrition app (non-)use. Interestingly, positive and negative consequences of use were only rarely addressed in previous reviews. For example, potential negative consequences of using nutrition apps such as feelings of guilt were only explicitly addressed in Lyzwinski et al. [27]. From a psychological point of view, however, anticipated or perceived positive and negative consequences for one's health, but also emotional consequences, are important precursors of engaging in the behavior (e.g., [76-78]). It could therefore be recommended to anticipate potential negative consequences of using an app to prevent them. At the same time, potential positive consequences of nutrition app use, including positive emotional consequences, e.g., increased well-being [79], should be emphasized more to promote use.

\section{Limitations and avenues for future research}

Some concerns regarding the findings of the review arise from the included studies. Several of the included studies investigated reasons for health app (non-)use more broadly. Although only studies were included that specifically addressed nutrition apps as one category of health apps, it 
could often not be determined whether an issue was raised in relation to nutrition apps or other categories of health apps. Thus, some barriers and facilitators might have been included in this review that did not refer to nutrition apps. Furthermore, most of the included studies did not provide information about anthropometrics or socioeconomic position of the participants, which makes it difficult to appraise generalizability of the findings. Finally, in many of the included studies, females were overrepresented, and two even focused exclusively on female participants [31,43]. While previous research suggests that nutrition app users are more likely to be female [15], including more male participants in future research would be desirable to address potential gender-specific needs (e.g., aiming to lose weight vs. aiming to gain muscle mass [80]) which might explain the lower adoption rates in males.

It is important to note that neither the present review nor previous reviews on barriers and facilitators for nutrition app use can provide insights into the relative importance of the barriers and facilitators for the decision (not) to use a nutrition app. While the number of studies in which a reason was mentioned could be used as an indicator, it might also reflect research questions and/ or questions and items used in the individual studies. Moreover, the grouping of barriers and facilitators into categories and clusters is somewhat arbitrary. Some categories in the framework might not be fully mutually exclusive, as for instance affinity for technology might also influence the perception of usability [81]. Notably, although Simblett et al. [23] only identified five categories of barriers and facilitators for the use of remote tracking technologies, many of the underlying barriers and facilitators could also be mapped onto the framework presented in this review. Further research is thus needed to gain insight into interrelations of the identified barriers and facilitators and their grouping based on empirical data as well as to determine their relative importance (see e.g. [24] for wearables).

Furthermore, barriers and facilitators might also differ between user groups, as has been highlighted in previous research on stage theories of behavior (e.g., [65,82]). Differences between user groups could not be disentangled in the present review as most studies reported barriers and facilitators for multiple user groups without indicating by which user group they were mentioned. One exception is the survey conducted by Murnane et al. [48], which showed that current users experienced positive consequences of health app use such as feeling more healthy and energized, while apps were abandoned because they did not function properly or lacked desired features. Furthermore, the importance of barriers and facilitators might also change while a nutrition app is 
used (see [83] for a discussion). As Baretta et al. [84] showed in the context of apps for physical activity promotion, some features like peer and coaching support might be more important for initial uptake, while e.g., proactive motivational features are more important for promoting continued use. Similarly, Sharpe et al. [28] highlighted that usability might be more important for sustained engagement with nutrition apps. Future research should therefore explicitly compare different user groups and stages to provide valuable insights on how to promote uptake and continued use of nutrition apps by specifically targeting relevant barriers and facilitators.

Finally, at least some of the barriers and facilitators identified in the present review might not be specific to the use of nutrition apps, but related to changing eating behavior independent of the mode of delivery. For instance, from some of the included publications, it did not become clear whether a lack of interest in nutrition apps referred to the app itself or changing the behavior. More research is therefore needed to disentangle these effects.

\section{Conclusions}

The present scoping review provides a comprehensive overview on barriers and facilitators for nutrition app use. A total of 326 barriers and facilitators were extracted from 28 publications and systematized in a framework with 21 sub-categories clustered in 12 categories. Four higher-order clusters were formed that subsume barriers and facilitators related to technology, the individual, their interactions, and the social environment. The resulting framework can be used to reduce barriers and specifically address facilitators of nutrition apps to increase and prolong their use.

\section{Acknowledgements}

This research was part of the SMARTACT project which was funded by the Federal Ministry of Education and Research (Grant 01EL1420A, granted to Britta Renner \& Harald Schupp). Furthermore, it was supported by a peer-mentoring grant from the Subdivision Health Psychology in the German Psychological Association awarded to LK and CA.

\section{References}

1. Kay M, Santos J, Takane M. mHealth: New Horizons for Health Through Mobile Technologies. Geneva, Switzerland: World Health Organization; 2011. ISBN: 9789241564

2. Pietsch J. The Mobile Health Market in Germany. Berlin, Germany: Germany Trade and Invest Gesellschaft für Außenwirtschaft und Standortmarketing mbH; 2017.

3. Aitken M, Clancy B, Nass D. The Growing Value of Digital Health. Parsippany, NJ: IQVIA 
Institute; 2017.

4. Omboni S, Caserini M, Coronetti C. Telemedicine and m-health in hypertension management: Technologies, applications and clinical evidence. High Blood Press Cardiovasc Prev 2016;23(3):187-196. PMID:27072129

5. Rehman H, Kamal AK, Morris PB, Sayani S, Merchant AT, Virani SS. Mobile health (mHealth) technology for the management of hypertension and hyperlipidemia: Slow start but loads of potential. Curr Atheroscler Rep 2017;19(3):12. PMID:28210974

6. Zhao J, Freeman B, Li M. Can mobile phone apps influence people's health behavior change? An evidence review. J Med Internet Res 2016;18(11):e287. PMID:27806926

7. Harrer M, Adam SH, Baumeister H, Cuijpers P, Karyotaki E, Auerbach RP, et al. Internet interventions for mental health in university students: A systematic review and meta-analysis. Int J Methods in Psychiatr Res 2019;28(2):e1759. PMID:30585363

8. Sama PR, Eapen ZJ, Weinfurt KP, Shah BR, Schulman KA. An evaluation of mobile health application tools. JMIR Mhealth Uhealth 2014;2(2):e19. PMID: 25099179

9. Aitken M, Gauntlett C. Patient Apps for Improved Healthcare: From Novelty to Mainstream. Parsippany, NJ: IMS Institute for Healthcare Informatics; 2013.

10. Wang Q, Egelandsdal B, Amdam GV, Almli VL, Oostindjer M. Diet and physical activity apps: Perceived effectiveness by app users. JMIR Mhealth Uhealth 2016;4(2):e33. PMID: 27056639

11. Schoeppe S, Alley S, Van Lippevelde W, Bray NA, Williams SL, Duncan MJ, et al. Efficacy of interventions that use apps to improve diet, physical activity and sedentary behaviour: A systematic review. Int J Behav Nutr Phys Act 2016;13(1):127. PMID:27927218

12. Villinger K, Wahl DR, Boeing H, Schupp HT, Renner B. The Effectiveness of App-Based Mobile Interventions on Nutrition Behaviors and Nutrition-Related Health Outcomes: A Systematic Review and Meta-Analysis. Obes Rev 2019;20:1465-1484. PMID:31353783

13. Number of smartphone users worldwide from 2016 to 2021 (in billions). Statista. https://www.statista.com/statistics/330695/number-of-smartphone-users-worldwide/. Published 2019. Accessed May 11, 2020.

14. Conway N, Campbell I, Forbes P, Cunningham S, Wake D. mHealth applications for diabetes: User preference and implications for app development. Health Informatics J 2016;22(4):1111-1120. PMID:26635324

15. König LM, Sproesser G, Schupp HT, Renner B. Describing the Process of Adopting Nutrition and Fitness Apps: Behavior Stage Model Approach. JMIR Mhealth UhHealth 2018;6(3):e55. PMID:29535078.

16. Ernsting C, Dombrowski SU, Oedekoven M, O'Sullivan JL, Kanzler M, Kuhlmey A, et al. Using smartphones and health apps to change and manage health behaviors: A populationbased survey. J Med Internet Res 2017;19(4):e101. PMID:28381394

17. Montagni I, Cariou T, Feuillet T, Langlois E, Tzourio C. Exploring digital health use and opinions of university students: Field survey study. JMIR Mhealth Uhealth 2018;6(3):e65. PMID:29549071

18. European Commission. eHealth Action Plan 2012-2020 - Innovative healthcare for the 21st century. Brussels, Belgium: Euopean Commission; 2012:1-14.

19. EU health policy. European Commission. https://ec.europa.eu/health/policies/overview_en. Accessed May 11, 2020.

20. Clawson J, Pater JA, Miller AD, Mynatt ED, Mamykina L. No longer wearing: Investigating the abandonment of personal health-tracking technologies on craigslist. Proceedings of the 2015 ACM International Joint Conference on Pervasive and Ubiquitous Computing; 2015 Sept 7-11; Osaka, Japan. New York, NY: ACM, 2015.

21. Hermsen S, Moons J, Kerkhof P, Wiekens C, De Groot M. Determinants for sustained use of an activity tracker: Observational study. JMIR Mhealth Uhealth 2017;5(10):e164. 
PMID:29084709

22. Eisenhauer CM, Hageman PA, Rowland S, Becker BJ, Barnason SA, Pullen CH. Acceptability of mHealth Technology for Self-Monitoring Eating and Activity among Rural Men. Public Health Nurs 2017;34(2):138-146. PMID:27757986

23. Simblett S, Greer B, Matcham F, Curtis H, Polhemus A, Ferrão J, et al. Barriers to and facilitators of engagement with remote measurement technology for managing health: Systematic review and content analysis of findings. J Med Internet Res 2018;20(7):e10480. PMID:30001997

24. Attig C, Franke T. Abandonment of personal quantification: A review and empirical study investigating reasons for wearable activity tracker attrition. Comput Human Behav 2020;102:223-237. doi:10.1016/j.chb.2019.08.025.

25. Cordeiro F, Bales E, Cherry E, Fogarty J. Rethinking the mobile food journal: Exploring opportunities for lightweight photo-based capture. Proceedings of the 33rd Annual ACM Conference on Human Factors in Computing Systems; 2015 April 18-23; Seoul, Republic of Korea. New York, NY: ACM, 2015.

26. Cordeiro F, Epstein DA, Thomaz E, Bales E, Jagannathan AK, Abowd GD, et al. Barriers and negative nudges: Exploring challenges in food journaling. Proceedings of the 33rd Annual ACM Conference on Human Factors in Computing Systems; 2015 April 18-23; Seoul, Republic of Korea. New York, NY: ACM, 2015.

27. Lyzwinski LN, Caffery LJ, Bambling M, Edirippulige S. Consumer perspectives on mHealth for weight loss: A review of qualitative studies. J Telemed Telecare 2018;24(4):290-302. PMID:28181859

28. Sharpe EE, Karasouli E, Meyer C. Examining factors of engagement with digital interventions for weight management: Rapid review. JMIR Res Protoc 2017;6(10):e205. PMID:29061557

29. Krebs P, Duncan DT. Health app use among US mobile phone owners: A national survey. JMIR Mhealth Uhealth 2015;3(4):e101. PMID:26537656

30. Jones M, Maddox J, Benavides-Espinoza C, Finnicum P. Use of Fitness and Nutrition Applications by College Students. Missouri Journal of Health, Physical Education, Recreation \& Dance 2017;27:63-71.

31. Eikey EV, Reddy MC. It's Definitely Been a Journey: A Qualitative Study on How Women with Eating Disorders Use Weight Loss Apps. Proceedings of the 2017 CHI conference on human factors in computing systems; 2017, May 6-11; Denver, CO. New York, NY: ACM; 2017.

32. Davis FD, Bagozzi RP, Warshaw PR. User acceptance of computer technology: A comparison of two theoretical models. Manage Sci 1989;35(8):982-1003. doi:10.1287/mnsc.35.8.982

33. Peters MD, Godfrey CM, Khalil H, McInerney P, Parker D, Soares CB. Guidance for conducting systematic scoping reviews. Int J Evid Based Healthc 2015;13(3):141-146. PMID:26134548

34. Munn Z, Peters MD, Stern C, Tufanaru C, McArthur A, Aromataris E. Systematic review or scoping review? Guidance for authors when choosing between a systematic or scoping review approach. BMC Med Res Methodol 2018;18(1):143. PMID:30453902

35. Tricco AC, Lillie E, Zarin W, O'Brien KK, Colquhoun H, Levac D, et al. PRISMA extension for scoping reviews (PRISMA-ScR): Checklist and explanation. Ann Intern Med 2018;169(7):467-73. PMID:30178033

36. Mayring P. Qualitative content analysis: Theoretical foundation, basic procedures and software solution. https://nbn-resolving.org/urn:nbn:de:0168-ssoar-395173. Published 2014. Accessed May 11, 2020.

37. Aljuraiban GS. Use of Weight-Management Mobile Phone Apps in Saudi Arabia: A WebBased Survey. JMIR Mhealth Uhealth. 2019;7(2):e12692. PMID:30794205 
38. Anderson K, Burford O, Emmerton L. Mobile health apps to facilitate self-care: A qualitative study of user experiences. PLoS One 2016;11(5):e0156164. PMID:27214203

39. Bhuyan SS, Lu N, Chandak A, Kim H, Wyant D, Bhatt J, et al. Use of mobile health applications for health-seeking behavior among US adults. J Med Syst 2016;40(6):153. PMID:27147516

40. Chan A, Kow R, Cheng JK. Adolescents' perceptions on smartphone applications (apps) for health management. J Mob Technol Med 2017;6(2):47-55. doi:10.7309/jmtm.6.2.6

41. Choe EK, Lee NB, Lee B, Pratt W, Kientz JA. Understanding quantified-selfers' practices in collecting and exploring personal data. Proceedings of the SIGCHI Conference on Human Factors in Computing Systems; 2014 April 26-May, 1; Toronto, Canada. New York, NY: ACM; 2014.

42. Dennison L, Morrison L, Conway G, Yardley L. Opportunities and challenges for smartphone applications in supporting health behavior change: Qualitative study. J Med Internet Res 2013;15(4):e86. PMID:23598614

43. Flaherty SJ, McCarthy MB, Collins AM, McAuliffe FM. A different perspective on consumer engagement: exploring the experience of using health apps to support healthier food purchasing. Journal of Marketing Management 2019;35(3-4):310-337. doi:10.1080/0267257X.2019.1576756

44. Gowin M, Cheney M, Gwin S, Franklin Wann T. Health and fitness app use in college students: A qualitative study. Am J Health Educ 2015;46(4):223-230. doi:10.1080/19325037.2015.1044140

45. Haithcox-Dennis M, Brinkley J, Richman A, DeWeese A, Byrd III JL. Mhealth on campus: Assessing undergraduates attitudes and utilization of mobile health applications. Glob J Health Educ Promot 2012;15(1):134-144.

46. Kwon M-W, Mun K, Lee JK, McLeod DM, D’Angelo J. Is mobile health all peer pressure? The influence of mass media exposure on the motivation to use mobile health apps. Convergence 2017;23(6):565-586. doi:10.1177/1354856516641065.

47. Lieffers JR, Arocha JF, Grindrod K, Hanning RM. Experiences and perceptions of adults accessing publicly available nutrition behavior-change mobile apps for weight management. J Acad Nutr Diet 2018;118(2):229-239. PMID:28625662

48. Murnane EL, Huffaker D, Kossinets G. Mobile health apps: Adoption, adherence, and abandonment. Adjunct Proceedings of the 2015 ACM International Joint Conference on Pervasive and Ubiquitous Computing and Proceedings of the 2015 ACM International Symposium on Wearable Computers; 2015 Sept 7-11; Osaka, Japan. New York, NY: ACM, 2015.

49. Oh J, Lee U. Exploring UX issues in Quantified Self technologies. Eighth International Conference on Mobile Computing and Ubiquitous Networking (ICMU); 2015 Jan 20-22; Hakodate, Japan. New York, NY: IEEE; 2015.

50. Peng W, Kanthawala S, Yuan S, Hussain SA. A qualitative study of user perceptions of mobile health apps. BMC Pub Health 2016;16(1):1158. PMID:27842533

51. Sarcona A, Kovacs L, Wright J, Williams C. Differences in eating behavior, physical activity, and health-related lifestyle choices between users and nonusers of mobile health apps. Am J Health Educ 2017;48(5):298-305. doi:10.1080/19325037.2017.1335630.

52. Solbrig L, Jones R, Kavanagh D, May J, Parkin T, Andrade J. People trying to lose weight dislike calorie counting apps and want motivational support to help them achieve their goals. Internet Interv 2017;7:23-31. PMID:28286739

53. Tang J, Abraham C, Stamp E, Greaves C. How can weight-loss app designers' best engage and support users? A qualitative investigation. Br J Health Psychol 2015;20(1):151-171. PMID:25130682

54. Warnick JL, Pfammatter A, Champion K, Galluzzi T, Spring B. Perceptions of health 
behaviors and mobile health applications in an academically Elite College population to inform a targeted health promotion program. Int J Behav Med 2019;26(2):165-174. PMID:30632092

55. West JH, Belvedere LM, Andreasen R, Frandsen C, Hall PC, Crookston BT. Controlling your “App"etite: How diet and nutrition-related mobile apps lead to behavior change. JMIR Mhealth Uhealth 2017;5(7):e95. PMID:28694241

56. Woldeyohannes HO, Ngwenyama OK. Factors influencing acceptance and continued use of mHealth apps. International Conference on HCI in Business, Government, and Organizations; 2017 July 9-14; Vancouver, Canada. Cham, Switzerland: Springer; 2017.

57. Yuan S, Ma W, Kanthawala S, Peng W. Keep using my health apps: Discover users' perception of health and fitness apps with the UTAUT2 model. Telemedicine and e-Health 2015;21(9):735-741. PMID:25919238

58. Zhou L, Bao J, Watzlaf V, Parmanto B. Barriers to and Facilitators of the Use of Mobile Health Apps From a Security Perspective: Mixed-Methods Study. JMIR Mhealth Uhealth 2019;7(4):e11223. PMID:30990458

59. Michie S, Richardson M, Johnston M, Abraham C, Francis J, Hardeman W, et al. The behavior change technique taxonomy (v1) of 93 hierarchically clustered techniques: Building an international consensus for the reporting of behavior change interventions. Ann Behav Med 2013;46(1):81-95. PMID:23512568

60. Abraham C, Michie S. A taxonomy of behavior change techniques used in interventions. Health Psychol 2008;27(3):379-387. PMID:18624603

61. Direito A, Dale LP, Shields E, Dobson R, Whittaker R, Maddison R. Do physical activity and dietary smartphone applications incorporate evidence-based behaviour change techniques? BMC Pub Health 2014;14(1):646. PMID:24965805

62. Turner-McGrievy GM, Wilcox S, Boutté A, Hutto BE, Singletary C, Muth ER, et al. The Dietary Intervention to Enhance Tracking with Mobile Devices (DIET Mobile) Study: A 6Month Randomized Weight Loss Trial. Obesity 2017;25(8):1336-1342. PMID:28600833

63. Dunn CG, Turner-McGrievy GM, Wilcox S, Hutto B. Dietary self-monitoring through calorie tracking but not through a digital photography app is associated with significant weight loss: The 2SMART pilot study-A 6-month randomized trial. J Acad Nutr Diet 2019;119(9):15251532. PMID:31155474

64. König LM, Renner B. Boosting healthy food choices by meal colour variety: results from two experiments and a just-in-time Ecological Momentary Intervention. BMC Pub Health 2019;19(1):975. PMID:31331299

65. Weinstein ND, Lyon JE, Sandman PM, Cuite CL. Experimental evidence for stages of health behavior change: The precaution adoption process model applied to home radon testing. Health Psychol 1998;17(5):445-453. PMID:9776003

66. Lally P, Gardner B. Promoting habit formation. Health Psycho Rev 2013;7(sup1):S137-S58. doi:10.1080/17437199.2011.603640.

67. Schembre SM, Liao Y, O'Connor SG, Hingle MD, Shen S-E, Hamoy KG, et al. Mobile ecological momentary diet assessment methods for behavioral research: Systematic review. JMIR Mhealth Uhealth 2018;6(11):e11170. PMID:30459148

68. Burke LE, Warziski M, Starrett T, Choo J, Music E, Sereika S, et al. Self-monitoring dietary intake: Current and future practices. J Ren Nutr 2005;15(3):281-290. PMID:16007557

69. Ziesemer K, König LM, Boushey CJ, Villinger K, Wahl DR, Butscher S, et al. Occurrence of and reasons for "missing events" in mobile dietary assessments: Results from three eventbased Ecological Momentary Assessment (EMA) studies. Manuscript under review. 2019.

70. Graham Thomas J, Bond DS, Ryder BA, Leahey TM, Vithiananthan S, Roye GD, et al. Ecological momentary assessment of recommended postoperative eating and activity behaviors. Surg Obes Relat Dis 2011;7(2):206-212. PMID:21130703 
71. Boushey C, Spoden M, Zhu F, Delp E, Kerr D. New mobile methods for dietary assessment: Review of image-assisted and image-based dietary assessment methods. Proc Nutr Soc 2017;76(3):283-294. PMID:27938425

72. Michie S, Abraham C, Whittington C, McAteer J, Gupta S. Effective techniques in healthy eating and physical activity interventions: A meta-regression. Health Psychol 2009;28(6):690701. PMID:19916637

73. Lyons EJ, Lewis ZH, Mayrsohn BG, Rowland JL. Behavior change techniques implemented in electronic lifestyle activity monitors: A systematic content analysis. J Med Internet Res 2014;16(8):e192. PMID:25131661

74. Perski O, Blandford A, West R, Michie S. Conceptualising engagement with digital behaviour change interventions: A systematic review using principles from critical interpretive synthesis. Transl Behav Med 2016;7(2):254-267. PMID:27966189

75. Noar SM, Benac CN, Harris MS. Does tailoring matter? Meta-analytic review of tailored print health behavior change interventions. Psychol Bull 2007;133(4):673-693. PMID:17592961

76. Schwarzer R. Modeling health behavior change: How to predict and modify the adoption and maintenance of health behaviors. Appl Psychol 2008;57(1):1-29. doi:10.1111/j.14640597.2007.00325.x.

77. Klusmann V, Musculus L, Sproesser G, Renner B. Fulfilled emotional outcome expectancies enable successful adoption and maintenance of physical activity. Front Psychol 2016;6:1990. PMID:26779095

78. Gellert P, Ziegelmann JP, Schwarzer R. Affective and health-related outcome expectancies for physical activity in older adults. Psychol Health 2012;27(7):816-828. PMID:21867397

79. Wahl DR, Villinger K, König LM, Ziesemer K, Schupp HT, Renner B. Healthy food choices are happy food choices: Evidence from a real life sample using smartphone based assessments. Sci Rep 2017;7(1):17069. PMID:29213109

80. Kelley CCG, Neufeld JM, Musher-Eizenman DR. Drive for thinness and drive for muscularity: Opposite ends of the continuum or separate constructs? Body Image. 2010;7(1):74-7. PMID:19944659

81. Franke T, Attig C, Wessel D. A personal resource for technology interaction: development and validation of the affinity for technology interaction (ATI) scale. Int J Hum Comput Interact 2019;35(6):456-467. doi:10.1080/10447318.2018.1456150

82. DiClemente CC, Carbonari JP, Velasquez MM. Alcoholism treatment mismatching from a process of change perspective. In: Watson R, editor. Alcohol abuse treatment. Totowa, NJ: The Humana Press; 1992, 115-142. ISBN: 978-1-4612-6726-3.

83. Graham AK, Lattie EG, Mohr DC. Experimental therapeutics for digital mental health. JAMA Psychiatry 2019;76(12):1223-1224. PMID:31433448

84. Baretta D, Perski O, Steca P. Exploring users' experiences of the uptake and adoption of physical activity apps: Longitudinal qualitative study. JMIR Mhealth Uhealth. 2019;7(2):e11636. PMID:30735143 


\section{Supplementary Files}




\section{Multimedia Appendixes}

Identified categories per publication.

URL: https://asset.jmir.pub/assets/af15f8e73e3426069f4561ee8897cf64.docx

Quotes extracted from publications including assigned category.

URL: https://asset.jmir.pub/assets/0296e3631fd1cabb08ab53920aacd098.xlsx

PRISMA checklist for scoping reviews.

URL: https://asset.jmir.pub/assets/cf3dd469063d1b91ee1f4d04217bf897.docx 